International Joumal of Biological Sciences
2010; 6(1):68-79

(c) Ivyspring International Publisher. All rights reserved

Research Paper

\title{
Keratin promoter based gene manipulation in the murine conducting airway
}

\author{
Stephen P. Malkoski ${ }^{1,3}{ }^{凶}$, Timothy G. Cleaver ${ }^{1}$, Shi-Long Lu², Jessyka G. Lighthall ${ }^{*}$, and Xiao-Jing Wang ${ }^{2,3 凶}$
}

1. Division of Pulmonary Sciences and Critical Care Medicine, University of Colorado Denver Health Sciences Center, Aurora CO, USA

2. Department of Otolaryngology, University of Colorado Denver Health Sciences Center, Aurora CO, USA

3. Department of Pathology, University of Colorado Denver Health Sciences Center, Aurora CO, USA

* Current Address: Department of Otolaryngology, Oregon Health \& Sciences University, Portland, OR, USA

$\triangle$ Correspondence to: Xiao-Jing Wang, MD, PhD, Departments of Pathology, Dermatology and Otolaryngology, 12800 E. 19th Avenue, Research 1 North, Room 5128, Box 8104 Aurora, CO 80045. Phone: 303-724-3001 Fax: 303-724-4730 Email: XJ.Wang@ucdenver.edu. Or Stephen Malkoski, MD, PhD, Division of Pulmonary Sciences and Critical Care Medicine and Pathology,12800 E. 19th Avenue, Research 2, C272, Aurora, CO 80045. Phone: 303-724-6058 Fax: 303-724-4730 Email: Stephen.Malkoski@ucdenver.edu

Received: 2009.12.10; Accepted: 2010.01.15; Published: 2010.01.20

\begin{abstract}
Systems capable of targeting genetic manipulations to keratin-positive airway basal cells are more poorly developed than systems targeting other airway epithelial cell populations and this has likely hindered development of animal models of diseases such as lung squamous cell carcinoma. Although keratin promoter driven-Cre recombinase constructs are potentially useful for targeting these cells, these constructs have substantially higher activity in the skin and oral epithelium than in the airways. We developed a method for delivering RU486, the conditional activator of Cre recombinase progesterone receptor (CrePR) fusion proteins to the lung and then examined the activity of three keratin-driven CrePR constructs in the conducting airways. We also developed a technique for survival bronchioalveolar lavage on non-ventilated animals to examine the effects of the acetone/oil vehicle required to deliver RU486 to the lung. K5CrePRI and KI4CrePRI constructs differ only in the keratin promoter used to target CrePRI expression while $\mathrm{K} 5 \mathrm{Cre}$ *PR contains a truncated progesterone receptor designed to reduce RU486-independent Cre activity. While all three constructs demonstrate RU486-inducible Cre activity in the conducting airways, both construct activity and tightness of regulation vary considerably. $\mathrm{K} 5 \mathrm{Cre}$ *PR is the most tightly regulated Cre driver making it ideal for targeting somatic mutations to the airway epithelia while $\mathrm{K} 5 \mathrm{CrePRI}$ and KI4CrePRI may be better suited to studying diseases of the conducting airways where gene targeting of keratin expressing cells and their derivatives is desired.
\end{abstract}

Key words: Keratins, Cre recombinase, basal cells

\section{Introduction}

The lung epithelium is comprised of numerous cell types and varies anatomically along the proximal to distal axis. Lung epithelial cells can be derived from different potential progenitor populations during normal development and injury-repair ${ }^{1-4}$. Basal cells appear to function as stem cells of the conducting airway by giving rise to other basal cells, Clara cells, and ciliated epithelial cells and by populating the conducting airways during development and after injury ${ }^{3,5-7}$. A variety of systems for targeting Cre re- 
combinase to the lung epithelium have been developed. Constitutive Cre recombinase expression has been targeted to Clara cells via the Clara cell secretory protein (CCSP) promoter, type II alveolar cells via the surfactant protein-C (SP-C) promoter, ciliated epithelial cells via the FoxJ1 promoter ${ }^{8-11}$, and less specifically by an adenoviral Cre recombinase vector 12 . Similarly, doxycycline-inducible Cre recombinase has been targeted to Clara cells via the CCSP promoter and alveolar type II cells via the SP-C promoter 13,14 . Introduction of oncogenic mutations with these Cre recombinase constructs has usually caused lung adenocarcinoma formation $12,15,16$ and lung squamous cell carcinomas formation has only rarely been reported 17 , potentially because these tumors are derived from keratin-positive airway basal cells not targeted by these approaches.

Both K14CreER ${ }^{\mathrm{T}} 18$ and K5CreER ${ }^{\mathrm{T} 2}{ }^{3}$ express tamoxifen-inducible Cre recombinase-estrogen receptor (ER) fusion proteins under the control of the keratin 14 or keratin 5 promoters and have previously been to lineage label airway basal cells 3,5,19. Although systemic tamoxifen treatment permitted temporal control of Cre expression in airway basal cells, it also caused Cre expression in both skin, buccal, and esophageal mucosa in at least one of these studies ${ }^{5}$. The lack of a tightly-regulated, basal cell targeting system and an optimized tracheal delivery mechanism of the activator of the Cre-recombinase fusion protein has likely limited the development of mouse models of basal cell specific diseases such as lung squamous cell carcinoma.

Our laboratory previously used a series of keratin-driven, RU486-inducible Cre recombinase-progesterone receptor (PR) fusion proteins for genetic manipulation in the skin and oral epithelium 20,21 . In these systems, keratin promoters are used to restrict the expression of the RU486 inducible Cre recombinase-PR fusion protein; upon treatment with the RU486 activator, the Cre recombinase-PR fusion protein translocates to the nucleus and excises sequences between Lox $\mathrm{P}$ sites, facilitating conditional genetic manipulation of target genes ${ }^{22}$. We were interested in determining whether these systems could be used to target keratin positive cells of the conducting airways. Because keratins are highly expressed in epidermis and oral epithelium, tracheal delivery of the lipid-soluble RU486 is required to limit gene manipulation to the conducting airway and minimize extrapulmonary Cre activity. In this series of experiments we first established a solvent system for delivering the lipid-soluble RU486 to the lung and then developed a technique for survival bronchioalveolar lavage (BAL) to assess the effects of using an acetone/oil vehicle in the lung. We evaluated the pulmonary and extrapulmonary Cre recombinase activity of three keratin-driven, RU486-inducible, CrePR constructs: K5CrePR1 ${ }^{23}$ and K14CrePR1 24 , which differ in the keratin promoter used to target CrePR1 expression, and K5Cre*PR ${ }^{20}$, which differs from $\mathrm{K} 5 \mathrm{CrePR} 1$ in that the progesterone element has been truncated to reduce RU486-independent Cre activity. Our experiments show that keratin promoter-directed CrePR expression coupled with direct tracheal delivery of the Cre recombinase activator is a useful way to target airway epithelial cells.

\section{Materials and Methods}

Maintenance and use of transgenic mice: Transgenic mice in a C57BL/ 6 background were maintained in specific pathogen free rooms in accordance with Oregon Health \& Sciences University or University of Colorado Denver policies on animal use and care; all procedures were approved by Institutional Animal Care and Use Committees. Keratin 5 CrePR1 (K5CrePR1) and Keratin 14 CrePR1 (K14CrePR1) mice express a truncated, RU486-inducible, progesterone receptor-Cre recombinase fusion protein under the control of a keratin 5 or keratin 14 promoter ${ }^{23,24}$. The Keratin 5 Cre*PR (K5Cre*PR) construct has an additional 16 amino acid deletion of the progesterone receptor (PR) designed to reduce RU486-independent Cre recombinase activity 20,25 . Rosa 26 reporter mice contain a lox-neomycin-lox sequence between a constitutive promoter and a $\beta$-galactosidase $(\beta$-gal) reporter ${ }^{26}$. Bigenic reporter mice were created by crossing K5CrePR1, K14CrePR1, K5Cre*PR mice with Rosa26 reporter mice to create mice heterozygous for both genes (Figure 1A). For each CrePR construct, one founder line that previously exhibited robust Cre activity in either the skin or oral mucosa was used for tracheal delivery experiments.

Experiments were performed on 3-4 week old mice and experimental groups contained at least 3 mice from multiple litters (individual experimental numbers are described in figure legends). K5CrePR1.Rosa26 mice were treated with $100 \mu \mathrm{g}$ of tracheal RU486 and $\beta$-gal activity assessed 7 days later. K14CrePR.Rosa26 mice were treated with two $500 \mu \mathrm{g}$ doses of tracheal RU486 given $5 \mathrm{~d}$ apart and $\beta$-gal activity evaluated 7 days later after the last RU486 dose. K5Cre*PR.Rosa26 mice were treated with two $500 \mu \mathrm{g}$ tracheal RU486 doses given $5 \mathrm{~d}$ apart then sacrificed either 7 days or 24 weeks after the last RU486 dose. Both male and female mice were used in all experiments; no gender differences were observed. 
A.

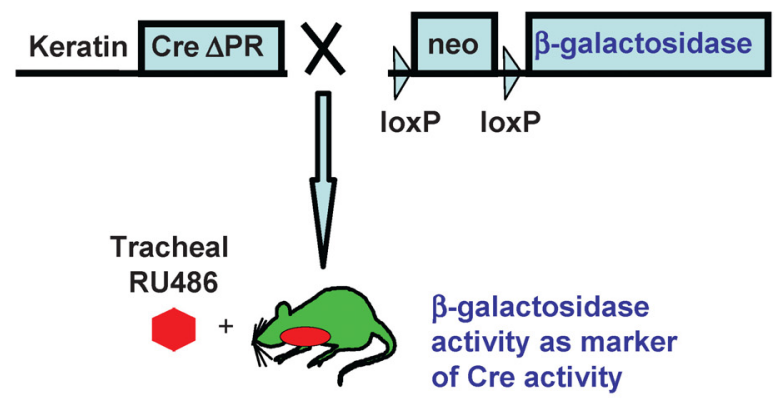

B.

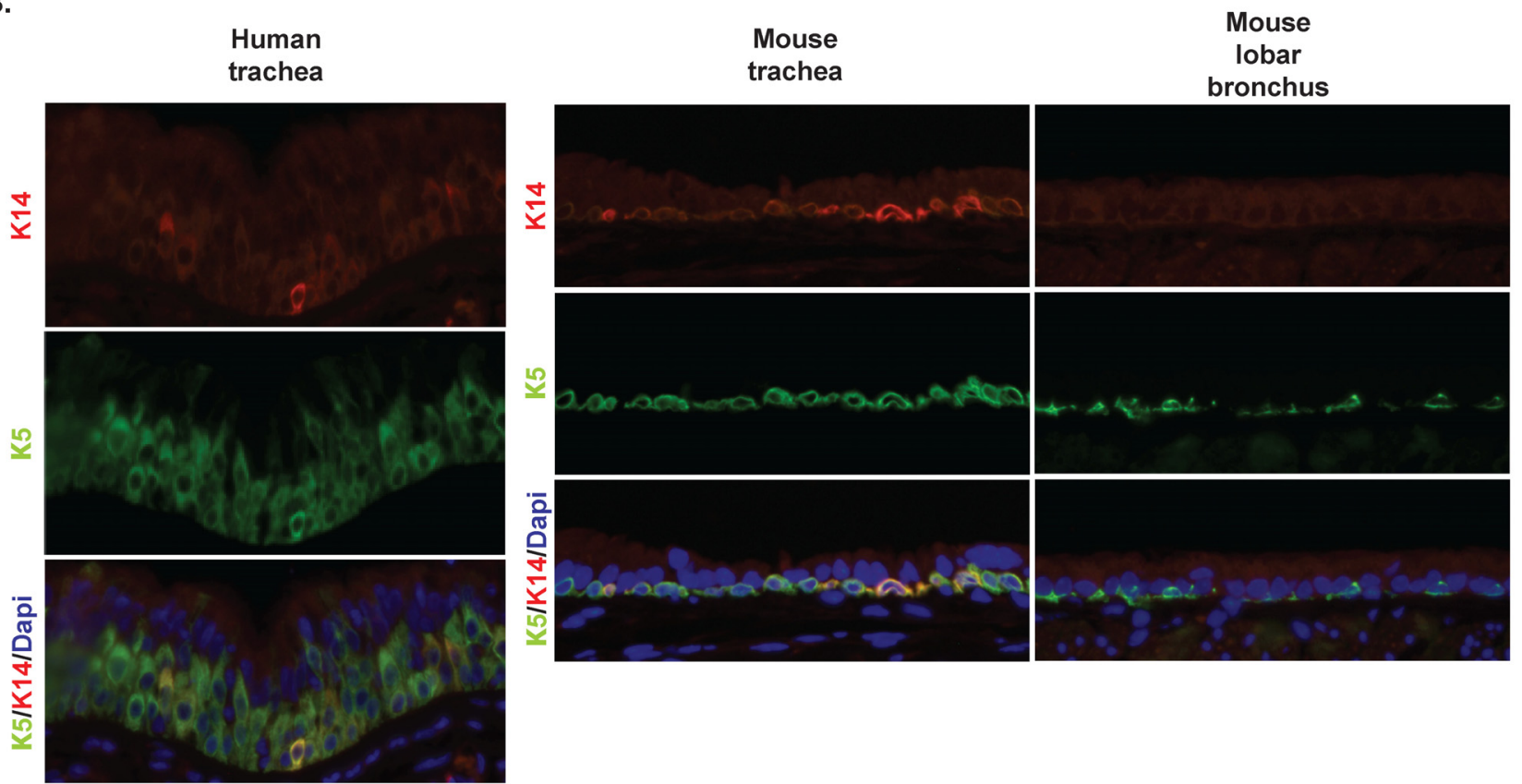

Figure I: (A) Keratin CrePR mice express a Cre recombinase-progesterone receptor (PR) fusion protein whose expression is restricted by a K5 or KI4 promoter. CrePR is inducible by the progesterone antagonist RU486 but not by endogenous progesterone. Upon induction with RU486, CrePR translocates into the nucleus and excises DNA sequences flanked by Lox P sites. Rosa26 reporter mice contain a lox P-neomycin-lox P sequence between a constitutive promoter and a $\beta$-gal reporter hence after Cre recombinase-mediated excision of the neomycin cassette $\beta$-gal is constitutively expressed and can be used to monitor Cre recombinase activity. (B) Immunofluoresence for K5/KI4 positive basal cells in the human and mouse trachea demonstrates the simpler conducting airway epithelium in the mouse. Minimal background immunostaining was observed when sections were incubated without primary antibody (not shown). The immunostaining pattern is consistent with marking $\mathrm{K} 5 / \mathrm{KI} 4$ positive basal cells. In the human trachea there are $23.4 \pm 1.4$ (mean $\pm \mathrm{SEM}$ ) $\mathrm{K} 5$ positive cells $/ 100 \mu \mathrm{m}$ epithelium and $5.6 \pm 0.9 \mathrm{KI} 4$ positive cells/ $100 \mu \mathrm{m}$ epithelium ( $<<0.05$ for $\mathrm{K} 5 \mathrm{vs}$. $\mathrm{K} / 4$ positive). In the mouse, there are $13.6 \pm 2.6 \mathrm{~K} 5$ positive cells $/ 100 \mu \mathrm{m}$ epithelium and $1.9 \pm 0.9 \mathrm{KI} 4$ positive cells/100 $\mu \mathrm{m}$ epithelium; in the axial bronchus there are $7.5 \pm 2.1 \mathrm{~K} 5$ positive cells $/ 100 \mu \mathrm{m}$ epithelium and $0.25 \pm 0.2 \mathrm{KI} 4$ positive cells $/ 100 \mu \mathrm{m}$ epithelium ( $\mathrm{p}<0.05$ for $\mathrm{K} 5$ positive vs. $\mathrm{KI} 4$ positive in both murine trachea and axial bronchus).

Tracheal intubation, drug delivery, and bronchioalveolar lavage (BAL): Tracheal intubation was performed as previously described 27 with minor modifications. Briefly, mice were anesthetized with mouse cocktail $(30 \mathrm{mg} / \mathrm{ml}$ ketamine; $3 \mathrm{mg} / \mathrm{ml}$ xylazine; $0.6 \mathrm{mg} / \mathrm{ml}$ acepromazine; $70 \mu \mathrm{l} / 20 \mathrm{~g}$ mouse i.p.) or $2-3 \%$ isoflurane and placed at a $60^{\circ}$ angle sus- pended by the top incisors. The trachea was transilluminated at the thoracic inlet and a bent chemical spatula used as a laryngoscope. Vocal cords were directly visualized and a 20 -gauge angiocath placed via a Seldinger technique. Correct tube positioning was confirmed by observing abdominal breathing with brief occlusion of the tube. RU486 (Biomol, Plymouth 
Meeting, PA) dissolved in various solvents was instilled into the trachea as detailed in Table 1. For 50\% organic solvent/saline trials 5 mice/group were used; for acetone/sesame oil trials, 9-11 mice/group were used. Dye delivery to evaluate anatomic precision of tracheal delivery was performed as follows. First, $10 \mu \mathrm{l}$ of $0.01 \%$ Evans blue dye in $10 \%$ acetone $/ 90 \%$ sesame oil solvent was drawn up into a previously calibrated length of PE10 tubing affixed to a blunted 25-gauge needle and a $1 \mathrm{~mL}$ syringe. After tracheal intubation, PE10 tubing was passed though the tracheal angiocath until slight resistance was met; dye was then instilled over 2-3 seconds. Animals were immediately extubated. Mice were sacrificed within 1 hour to evaluate dye distribution in the in the lungs.

Survival bronchioalveolar lavage (BAL) was performed using isoflurane anesthesia and a $4 \mathrm{~cm}$ length of PE20 tubing attached to a $1 \mathrm{~mL}$ syringe containing $200 \mu \mathrm{l}$ of normal saline. After tracheal intubation, PE20 tubing was passed through the catheter until slight resistance was encountered. Sterile normal saline $(200 \mu \mathrm{l})$ was instilled over 5 seconds and immediately withdrawn and placed on ice. Following BAL, mice were extubated and returned to their cages to recover. BAL fluid was cytospun onto slides (5min at 500rpm) then stained with either Diff Quik (Fisher Scientific, Pittsburg, PA) or Oil Red O (Sigma, St Louis, MO). Differential cell counts were determined by morphological criteria and differences between groups compared by unpaired t-tests.

Immunofluoresence (IF) and Immunohistochemistry (IHC): IF and IHC were performed as previously described ${ }^{21}$. After heat-mediated antigen retrieval in $10 \mathrm{mM}$ citrate for $10 \mathrm{~min}$, sections were incubated overnight at $4^{\circ} \mathrm{C}$ with antibodies against Keratin 5 (1:500 Abcam, Cambridge, MA), K14 (1:1000, Abcam), p63 (1:50 Santa Cruz Biotechnology, Santa Cruz, CA), or CCSP (1:2000 Upstate, Billerica, $\mathrm{MA})$; antigen was detected by incubation with species-appropriate, fluorochrome- conjugated secondary antibody (1:100 Invitrogen, Carlsbad, CA) or biotinylated secondary antibody (1:100, Vector Laboratories, Burlingame, $\mathrm{CA}$ ).

$\beta$-gal staining: $\beta$-gal activity was evaluated as previously described 5. After euthanasia, whole organs were removed and fixed for $20 \mathrm{~min}$ in $4 \%$ parafomaldehyde at room temperature, rinsed in PBS, then incubated at $37^{\circ} \mathrm{C}$ for $4-16 \mathrm{~h}$ in X-gal staining solution $\quad\left[2 \mathrm{mM} \quad \mathrm{MgCl}_{2}, \quad 5 \mathrm{mM} \quad \mathrm{K}_{4} \mathrm{Fe}(\mathrm{CN})_{6}, \quad 5 \mathrm{mM}\right.$ $\mathrm{K}_{3} \mathrm{Fe}(\mathrm{CN})_{6}, 0.02 \%$ NP40, 0.01\% sodium deoxycholate, $1 \mathrm{mg} / \mathrm{ml}$ 5-bromo-4-chloro-3-indolyl- $\beta$-D-galactopyranoside (X-gal)], then postfixed in $4 \%$ paraformaldehyde overnight at $4^{\circ} \mathrm{C}$. $\beta$-gal activity in whole organs was photographed through a Nikon SMZ1000 dissecting microscope with a Nikon DS Ri1 camera using Nikon Elements software. $\beta$-gal stained organs were paraffin embedded, sectioned, and counterstained with nuclear fast red or with immunohistochemistry as described above. The recombination frequency was determined by counting the number of $\beta$-gal positive cell per $100 \mu \mathrm{m}$ tracheal epithelium in at least 3 separate tracheal sections.

Human tracheal samples: Normal human tracheal sections from lung transplant donors were provided by Dr. David Jacoby (Oregon Health \& Sciences University, Portland, OR) with approval of the Institutional Review Board. Three human tracheal samples were evaluated.

\section{Results}

Keratin expression in murine conducting airways: In human airways, the number of keratin positive basal cells is $\sim 30 \%$ in the large ( $>4 \mathrm{~mm}$ ) airways but falls to $6 \%$ in airways $<0.5 \mathrm{~mm}{ }^{28}$. Similarly, basal cells (identified by histology) comprise $\sim 18 \%$ of airway epithelial cells in the mouse trachea but $<3 \%$ of airway epithelial cells in the axial bronchus and distal airway ${ }^{29}$. We confirmed a similar distribution of K5 positive cells in the human trachea, mouse trachea, and mouse axial bronchus. Interestingly, K14 expression appeared restricted to a subset of $\mathrm{K} 5$ positive cells in all three conducting airway epithelia (Figure 1B). Compared to the human trachea, in the mouse trachea, K5 positive basal cells are less numerous and restricted to a single cell layer. Though clearly more numerous in the trachea, basal cells (identified by $\mathrm{K} 5$ staining) are still detectable in the lobar bronchus (Figure 1B). These observations support the hypothesis that keratin promoters can target gene manipulation to the murine conducting airways.

Optimization of tracheal RU486 delivery: Because keratins are expressed in many epithelial tissues including the skin, oral mucosa, and urogential mucosa, targeting keratin positive airway cells without targeting keratin positive cells in other epithelial layers requires both tracheal delivery of the conditional gene activator and a system with minimal activator-independent Cre recombinase activity. Because RU486, the conditional activator of the truncated progesterone receptor-Cre recombinase, has minimal aqueous solubility, we investigated alternative solvents for tracheal RU486 delivery (Table 1). The goal of these experiments was to determine whether animals would tolerate tracheal delivery of the $50 \%$ organic solvent mixtures necessary to dissolve RU486. While tracheal instillation of $50 \mu 1$ normal saline is well tolerated, the organic solvents required to dissolve RU486 were associated with unacceptably high mor- 
tality upon tracheal instillation. In addition, these solvent combinations were only capable of delivering $10 \mu \mathrm{g}$ of RU486. When RU486 was dissolved in acetone (at $100 \mu \mathrm{g} / \mu \mathrm{l}$ ) then diluted 1:10 with sesame oil, $10 \mu \mathrm{l}$ of $10 \%$ acetone $/ 90 \%$ sesame oil was well tolerated and $100 \mu \mathrm{g}$ RU486 could be delivered with a $11 \%$ mortality; when the volume was increased to $20 \mu \mathrm{l}$, mortality increased to $22 \%$. Because respiratory depression secondary to ketamine/xylazine anasthesia was though to contribute to mortality after tracheal instillation of a viscous vehicle, we tested whether a shorter acting inhalational anesthetic would reduce procedural mortality and found that with isoflurane anesthesia, a higher concentration of acetone $(20 \%)$ and larger volume $(25 \mu \mathrm{l})$ facilitated the tracheal delivery of up to $500 \mu \mathrm{g}$ of RU486 without appreciable mortality.

Table I: Mortality of various solvents evaluated for tracheal RU486 delivery. Mortality is expressed as both a percentage and raw number. For all vehicle combinations except $20 \%$ acetone $/ 80 \%$ sesame oil, ketamine anesthesia was used. With $50 \%$ DMSO/50\% saline and $50 \%$ acetone $/ 50 \%$ saline, death occurred immediately upon instillation while with $50 \%$ ethanol $/ 50 \%$ saline, death occurred $3-5$ days later. With the acetone/oil vehicles, all observed mortality occurred with instillation.

\begin{tabular}{llll}
\hline Vehicle & Volume & Mortality & RU486 \\
\hline Normal saline & $50 \mu \mathrm{l}$ & $0 \%(0 / 5)$ & $0 \mu \mathrm{g}$ \\
\hline $50 \%$ DMSO/50\% saline & $10 \mu \mathrm{l}$ & $100 \%(5 / 5)$ & $10 \mu \mathrm{g}$ \\
\hline $50 \%$ acetone/ $50 \%$ saline & $10 \mu \mathrm{l}$ & $100 \%(5 / 5)$ & $10 \mu \mathrm{g}$ \\
\hline $50 \%$ ethanol/50\% saline & $10 \mu \mathrm{l}$ & $80 \%(4 / 5)$ & $10 \mu \mathrm{g}$ \\
\hline $10 \%$ acetone/90\% oil & $10 \mu \mathrm{l}$ & $11 \%(1 / 9)$ & $100 \mu \mathrm{g}$ \\
\hline $10 \%$ acetone/90\% oil & $20 \mu \mathrm{l}$ & $22 \%(2 / 9)$ & $200 \mu \mathrm{g}$ \\
$\begin{array}{l}20 \% \text { acetone/80\% oil } \\
\text { (isoflurane anesthesia) }\end{array}$ & $25 \mu \mathrm{l}$ & $0 \%(0 / 11)$ & $500 \mu \mathrm{g}$ \\
\hline
\end{tabular}

To assess the anatomic precision of tracheal delivery, we instilled Evans blue dye into either the trachea (Figure 2A) or the right main bronchus (Figure $2 \mathrm{~B}$ ) as described in the Methods. With tracheal dye instillation, minimal staining was observed in the esophagus while marked staining of the trachea and lung occurred (Figure 2A), demonstrating that this technique does not result in significant inadvertent exposure of other keratin expressing tissues (e.g., the oropharynx). The ability to deliver this vehicle almost exclusively to one lung (Figure 2B) shows the feasibility of unilaterally targeting lung cancer inducing genetic alterations to either prevent premature death from tracheal obstruction or to allow additional time for lesions to progress to full malignancy or metastasize.

Clearance of Lipid Laden Macrophages After
Tracheal Delivery of Sesame Oil: One concern regarding the use of sesame oil to deliver RU486 to the lungs is the development of a lipoid pneumonia characterized by an abundance of lipid-laden macrophages (LLMs), as occurs with chronic lipid aspiration in humans ${ }^{30}$. To examine this possibility we evaluated LLM clearance from the BAL fluid using survival BAL as described in the Methods. Using isoflurane anesthesia, survival BAL can be performed on non-ventilated mice with a mortality of $<8 \%$ and a return of approximately $50 \%$ of the lavage fluid volume. Mice appeared normal within hours after survival lavage, without evidence of respiratory distress, tachypnea, or huddled posture. All procedural mortality was immediate suggesting asphyxia as the cause of death; no delayed mortality consistent with pneumonia was observed. On untreated animals survival BAL specimens are $>95 \%$ macrophages, however, $3 \mathrm{~d}$ after tracheal instillation of $20 \mu \mathrm{l}$ of $20 \%$ acetone/ $80 \%$ sesame oil, $50 \%$ of cells recovered by BAL are LLM, while $45 \mathrm{~d}$ after instillation, $8 \%$ of cells recovered by BAL are LLM (Figure 2C). Tracheal instillation of acetone/sesame oil causes a modest inflammatory response with neutrophils comprising $26 \%$ of BAL cells $3 \mathrm{~d}$ after tracheal instillation; however, this inflammation resolves almost completely $45 \mathrm{~d}$ post instillation. Despite the short-term inflammatory response demonstrated by BAL, there was no obvious inflammation or morphological abnormalities in either the conducting airways or lung parenchyma at 5 days, 5 weeks, or 5 months after instillation of RU486 in acetone/sesame oil vehicle (Supplemental Figure S1). In addition to describing a technique for repeatedly sampling the lower airways in non-ventilated mice, these observations document LLM clearance after instillation of a lipid-based RU486 carrier.

Cre recombinase activity in K5CrePR1.Rosa26 mice: We initially examined K5CrePR1 activity in the trachea because this construct exhibited the most robust activity in both skin and oral mucosa 20, 21, 23 .

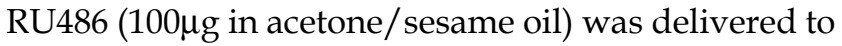
the trachea then Cre activity was detected by $\beta$-gal staining 7 days later. Tracheal dosing and timing was chosen based on doses previously used for gene manipulation in other keratin expressing epithelial tissues 20, 21, 23. Although vehicle treated K5CrePR1.Rosa26 mice exhibited a modest amount of RU486-independent activity (Figure 3A, middle panels), RU486 caused a significant induction in tracheal Cre activity as evidenced by the extensive $\beta$-gal staining in both whole mount and histology sections (Figure 3A, right panels). To characterize the airway cells targeted by $\mathrm{K} 5 \mathrm{CrePR} 1$, tracheal sections from RU486 treated K5CrePR1.Rosa26 mice were immu- 
nostained for the basal cell markers, $\mathrm{K} 5$ and p63, and the Clara cell marker, CCSP. We found that $\beta$-gal staining co-localized with $\mathrm{K} 5$ and p63 immunostaining confirming that airway basal cells are targeted by K5CrePR1. That $\beta$-gal staining also co-localized with

A.

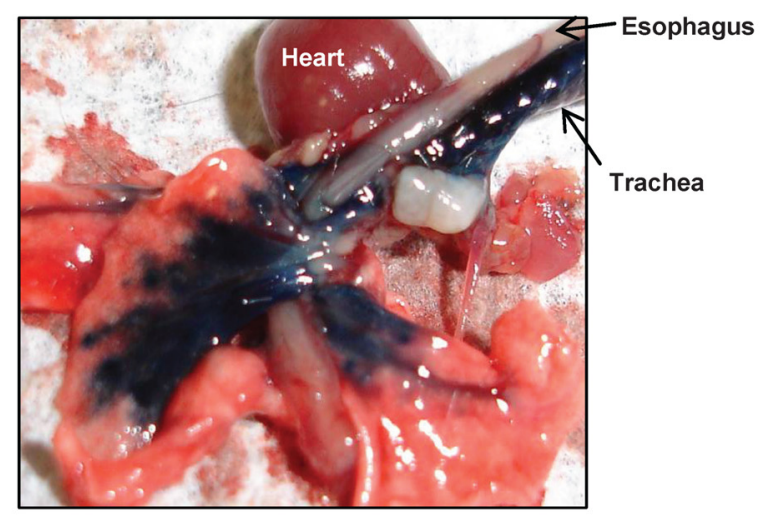

CCSP positive cells suggests either that previously targeted basal cells have differentiated into Clara cells 5,19 or that the K5CrePR1 construct also targets Clara cells.
B.

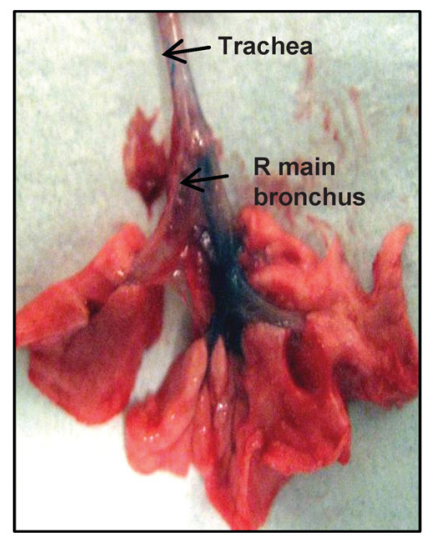

c.
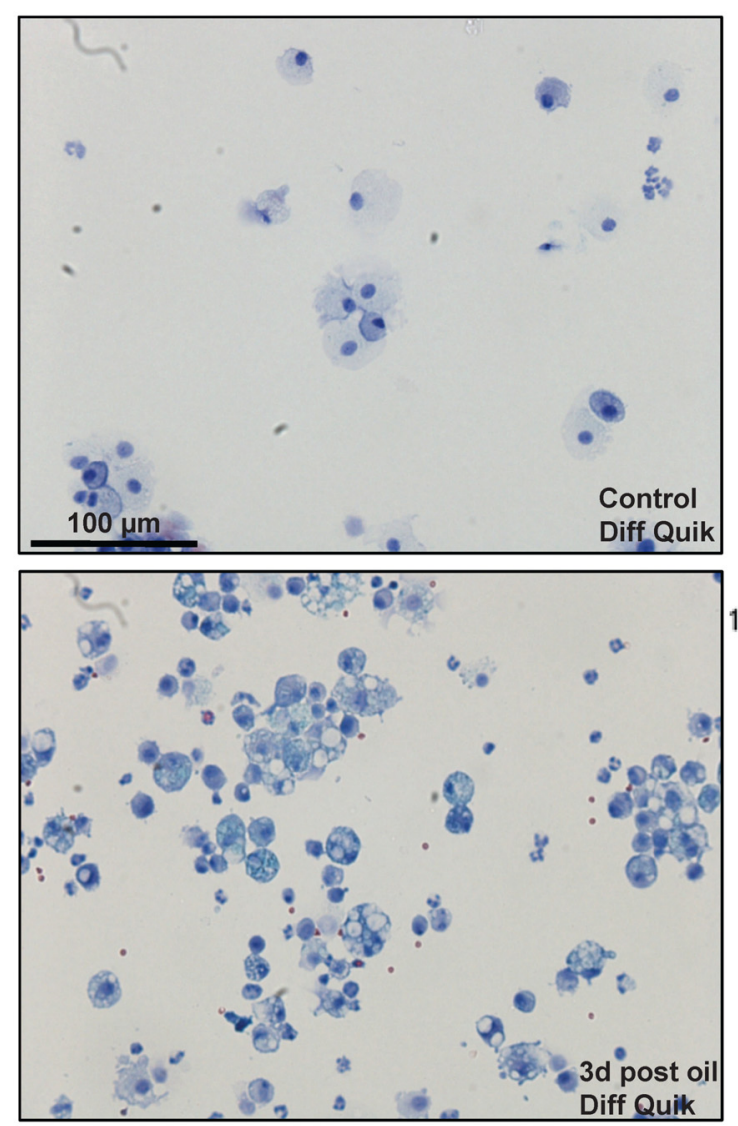
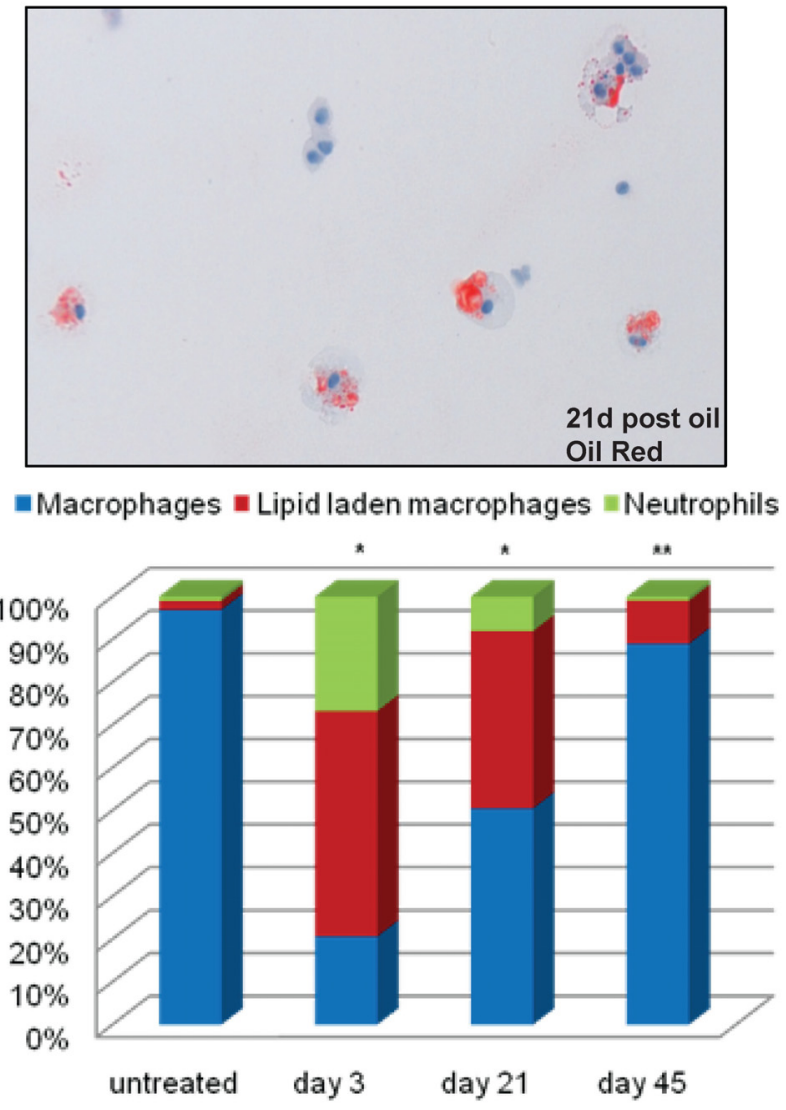

Figure 2: (A) Excised heart-lung block after tracheal instillation of $10 \mu 10.01 \%$ Evans blue dye in $10 \%$ acetone/90\% sesame oil showing staining in the trachea and lung but not in the esophagus. (B) Evans blue dye instillation to the right lung was performed as described in the methods. (C) Recovery of LLM by survival BAL after tracheal instillation of acetone/sesame oil. Untreated 8-12 week old animals $(n=12)$ underwent BAL with $200 \mu l$ normal saline as described in Methods. Approximately $95 \%$ of recovered cells were macrophages. A separate group of mice $(n=15)$ was treated with $20 \mu l$ of $20 \%$ acetone $/ 80 \%$ sesame oil on day 0 then underwent survival BAL on either day 3,21 , or 45 ( $n=5$ for each time point). Three days after treatment there was marked increase in both LLM and neutrophils (bottom left), however, these changes largely resolved by day $45 . * p<0.05$ vs. untreated control for all cell types; ${ }^{* *} p<0.05$ vs. days 3 and 21 for all cell types. 
A.

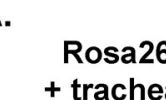

+ tracheal

RU486

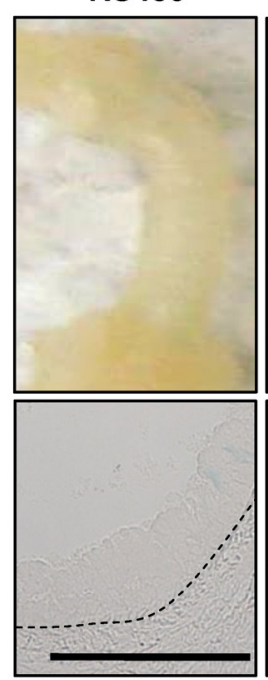

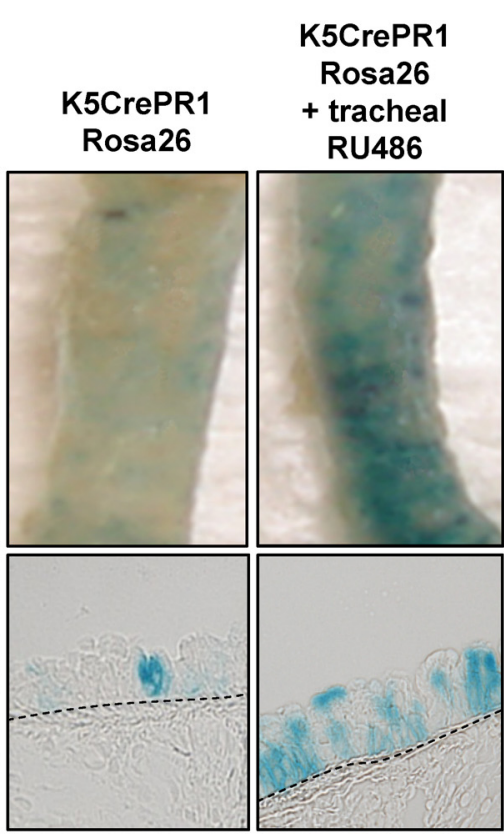

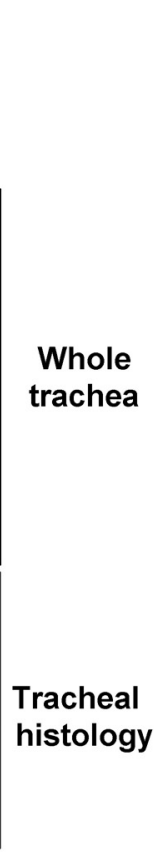

B.

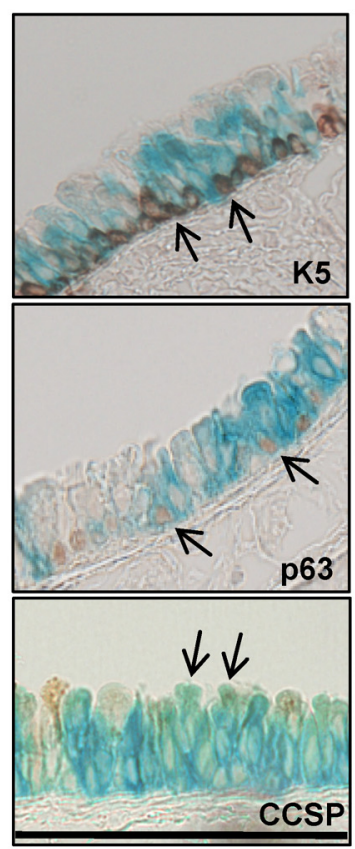

Figure 3: Cre activity after tracheal RU486 in K5CrePRI.Rosa26 mice. (A) K5CrePRI.Rosa26 mice were treated with $100 \mu g$ tracheal RU486 and $\beta$-gal activity assessed 7d later. No $\beta$-gal staining was observed in Cre negative monogenic littermates while RU486-inducible Cre activity was seen in K5CrePRI.Rosa26 animals. Representative images are shown; 3-4 mice/group were analyzed for $\beta$-gal expression. The dashed line delineates the epithelial basement membrane; the scale bar represents $100 \mu \mathrm{m}$. Untreated K5CrePRI.Rosa26 mice had $5.3 \pm 1.0$ (mean \pm SEM) recombinant cells/I00 $\mu \mathrm{m}$ tracheal epithelium while RU486-treated mice had $14.5 \pm 4.2$ recombinant cells/l00 $\mu \mathrm{m}$ tracheal epithelium $(\mathrm{p}=0.05)$. (B) Co-localization of $\beta$-gal staining with K5, p63, and CCSP expression in K5CrePRI.Rosa26 mice treated with tracheal RU486. Arrows indicate co-localization of $\beta$-gal and immunohistochemical staining in basal cells (K5+ and p63+) and Clara cells (CCSP+).

Inducible Cre recombinase activity in K14CrePR1.Rosa26 mice: While K5CrePR1 can be activated in the trachea, as animals age, substantial RU486-independent activity occurs in many keratin expressing tissues (not shown), however this activity is typically less than RU486-induced activity ${ }^{21,31}$. We evaluated whether K14CrePR1 could direct Cre activity to the trachea and whether K14CrePR1 also exhibited RU486-independent Cre activity. K14CrePR1.Rosa26 mice were treated with tracheal

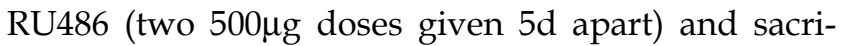
ficed $7 \mathrm{~d}$ after the last RU486 dose to evaluate $\beta$-gal activity. A higher RU486 dose was used in experiments with both K14CrePR and K5Cre*PR as lower doses appeared relatively ineffective in inducing Cre activity in pilot experiments (not shown). The RU486-independent activity of K14CrePR1 was markedly less than that of K5CrePR, and although $\beta$-gal positive cells could be seen on vehicle-treated whole mount tracheal sections, it was difficult to detect these cells in histology sections. After RU486 treatment, however, discrete areas of $\beta$-gal staining were clearly seen in the whole mount trachea and were more apparent when the trachea was opened longitudinally (Figure 4A). In addition, distinct $\beta$-gal staining was occasionally seen in the lung parenchyma (Figure 4A, right); consistent with either the presence of rare basal cells in the more distal airways or sporadic targeting of more distal Clara cells. Immunostaining for $\mathrm{K} 5$, p63, and CCSP again colocalized with $\beta$-gal staining (Figure $4 \mathrm{~B}$ ) suggesting that K14CrePR1 targets basal cells and their progeny. Compared to K5CrePR1, K14CrePR1 exhibits both less RU486-independent and RU486-induced Cre activity, perhaps because $\mathrm{K} 14$ is only expressed in a subset of K5 positive basal cells (see Figure 1B).

$\mathrm{K} 5 \mathrm{Cr} \mathrm{e}^{* \mathrm{PR}}$ is tightly regulated with inducible and persistent Cre activity: The $\mathrm{K} 5 \mathrm{Cre}{ }^{*} \mathrm{PR}$ construct is analogous to K5CrePR1 except for a 16 amino acid deletion of the truncated progesterone receptor designed to reduce RU486-independent Cre recombinase activity ${ }^{25}$. This construct has been used in mouse models of both skin and oral cancer ${ }^{21,32}$. We wanted 
to determine whether this construct would be active in the conducting airways and whether it would exhibit less RU486-independent activity than K5CrePR1 or K14CrePR1. K5Cre*PR.Rosa26 mice were treated

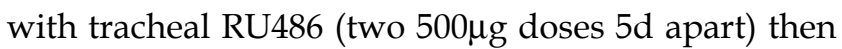
sacrificed $7 \mathrm{~d}$ after the last RU486 dose. We observed minimal RU486-independent activity in the airways (Figure 5A) or in other keratin expressing tissues (not shown); however, RU486 was still capable of inducing tracheal Cre activity (Figure 5A). Although K5Cre*PR has less tracheal Cre activity than K5CrePR1 or K14CrePR1, Cre activity still localized to K5/p63 positive basal cells and Clara cells (Figure 5B). Be- cause $\mathrm{K} 5 \mathrm{Cre}{ }^{*} \mathrm{PR}$ was the most tightly regulated construct tested, we assessed whether Cre-mediated gene recombination persisted after RU486 treatment and found increased $\beta$-gal activity in K5Cre*PR.Rosa26 animals 24 weeks after tracheal RU486 treatment compared to either vehicle treated animals or animals assessed $7 \mathrm{~d}$ after treatment (Figure 5C). Again, $\beta$-gal staining colocalized with $\mathrm{K} 5$, p63, and CCSP immunostaining, however, at this time point a larger fraction of tracheal epithelial cells had been labeled, consistent with the hypothesis that recombination initially occurred in a basal cell progenitor.

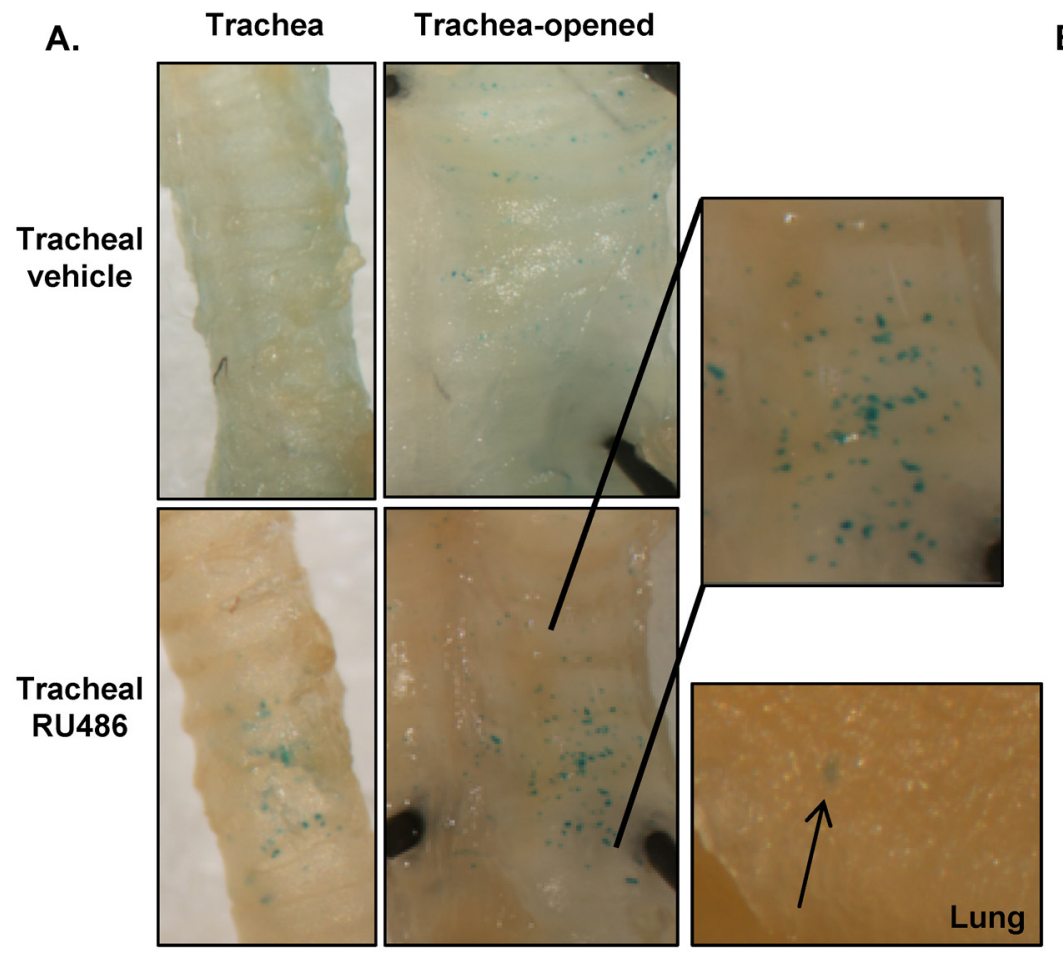

B.
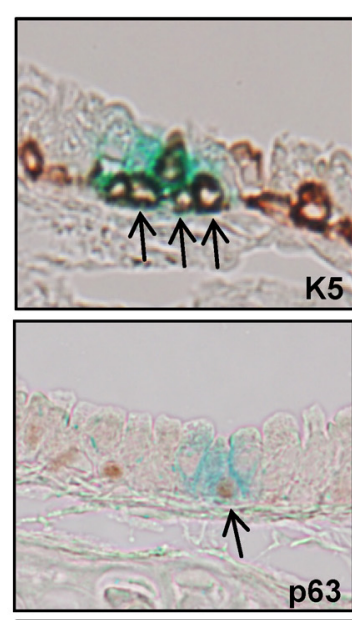

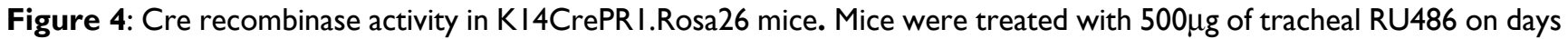
$I$ and 5 and then assessed for $\beta$-gal activity $7 \mathrm{~d}$ after the last RU486 dose. (A) Cre activity was induced in the trachea and was localized to discrete areas in the tracheal epithelium, however, $\beta$-gal staining was also occasionally observed in the lung parenchyma, consistent with rare basal cells in more distal airways. Representative images are shown; 3-8 mice per group were analyzed for $\beta$-gal expression. (B) Immunohistochemistry for $\mathrm{K5}$, $\mathrm{p} 63$, and CCSP colocalizes with $\beta$-gal staining in KI4CrePRI.Rosa26 mice treated with tracheal RU486. The scale bar represents $50 \mu \mathrm{m}$; arrows indicate co-localization of $\beta$-gal with K5, p63, and CCSP immunohistochemical staining; $1.3 \pm 0.6$ recombinant cells/ $/ 00 \mu \mathrm{m}$ tracheal epithelium were observed in RU486 treated animals compared to $0.1 \pm 0.07$ recombinant cells $/ 100 \mu \mathrm{m}$ tracheal epithelium in vehicle treated animals $(p<0.05)$. 


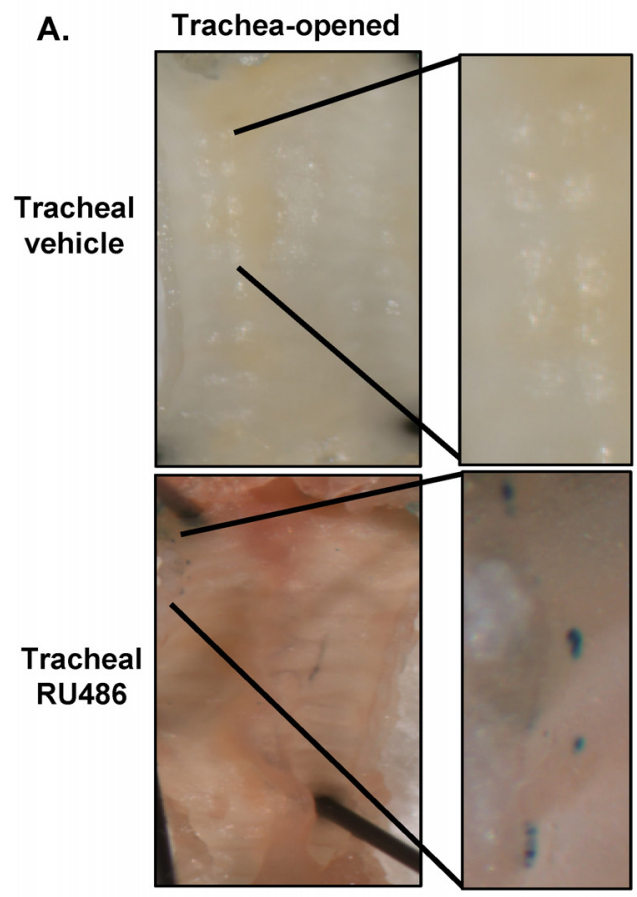

B.

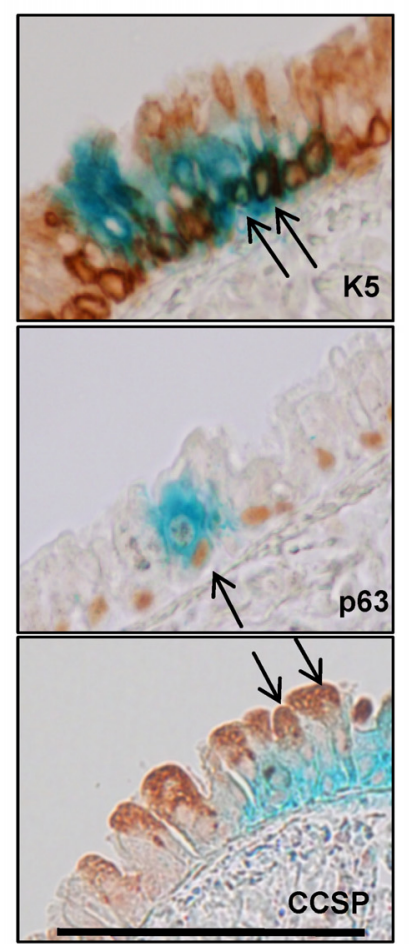

C.

Trachea-opened

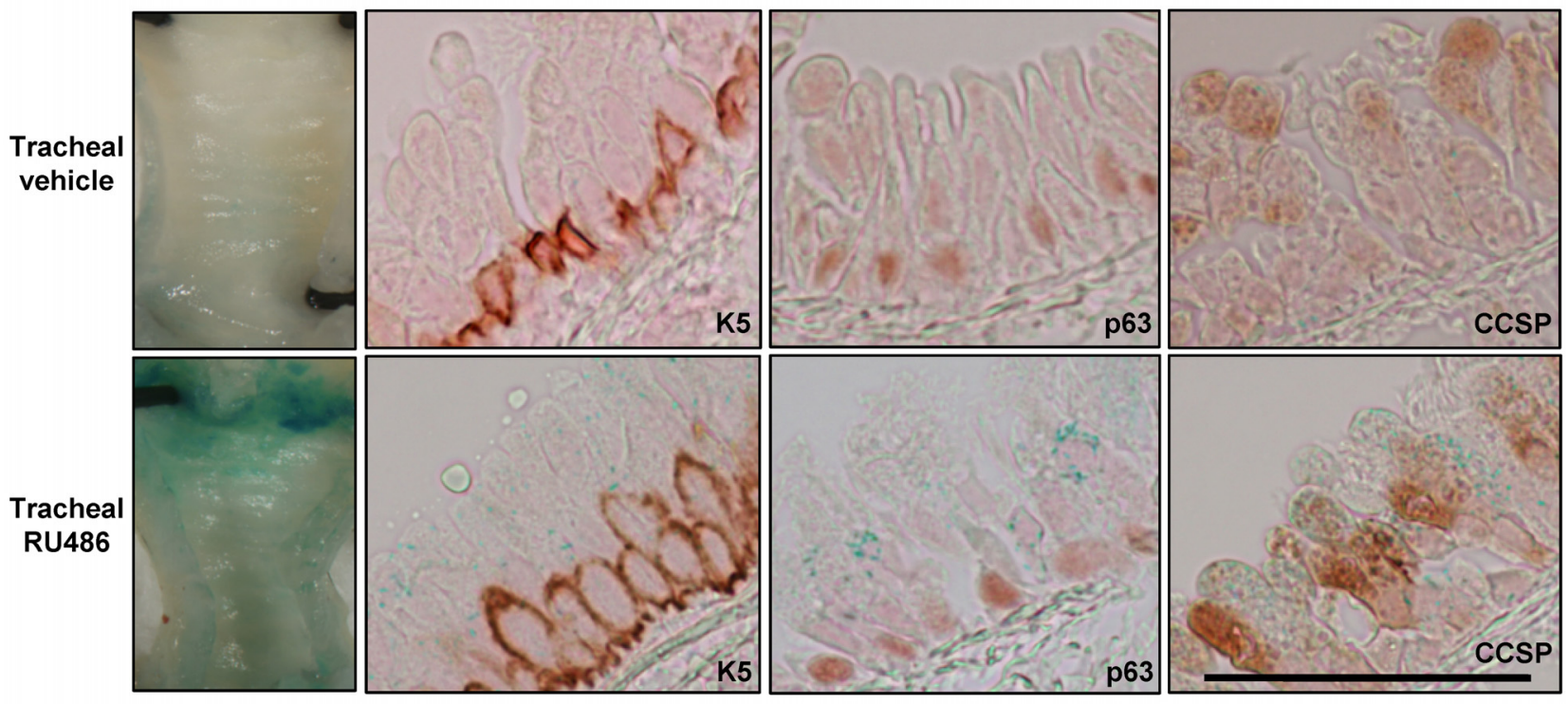

Figure 5: Inducible and persistent Cre recombinase activity in K5Cre*PR.Rosa26 mice after tracheal RU486. (A) In mice treated with $500 \mu \mathrm{g}$ of tracheal RU486 on days I and 5 and assessed for $\beta$-gal activity $7 \mathrm{~d}$ later, discrete areas of $\beta$-gal activity were seen in the trachea. $\beta$-gal staining was not observed in the tongue or esophagus of RU486-treated animals (not shown) or in vehicle treated animals. Representative images are shown; 5-10 mice per group were analyzed. (B) $\beta$-gal activity colocalized with K5, p63, and CCSP immunostaining in the trachea of RU486-treated K5Cre*PR.Rosa26 mice. The scale bar represents $50 \mu \mathrm{m}$; arrows indicate co-localization of $\beta$-gal and immunohistochemical staining; $0.3 \pm 0.16$ recombinant cells $/ 100 \mu \mathrm{m}$ tracheal epithelium were observed in RU486 treated mice; recombinant cells could not be found in the tracheal epithelium of vehicle treated animals. (C) Persistent tracheal Cre recombinase activity in K5Cre*PR.Rosa26 mice 24 weeks after tracheal RU486. Although $\beta$-gal staining was weaker compared to earlier time points, it still colocalized with K5, p63, and CCSP staining and was not detected in vehicle-treated tissues (top panel). The scale bar represents $50 \mu \mathrm{m} ; 17.5 \pm 0.8$ recombinant cells/ $/ 00 \mu \mathrm{m}$ tracheal epithelium were observed ( $\mathrm{p}<0.05$ vs. $7 \mathrm{~d}$ after RU486). 


\section{Discussion}

This study describes how a series of keratin-driven, conditionally activated Cre recombinase constructs in combination with tracheal delivery of the Cre inducer can be used for gene manipulation in murine conducting airway. While all three keratin CrePR constructs target the conducting airway epithelium, there are important differences that will influence use of these constructs. K5CrePR1 provides robust Cre activity and genetic recombination in most of epithelial cells of the murine trachea but has substantial ligand-independent activity in the trachea as well as in the skin and oral mucosa that could limit the utility of this construct if genetic recombination outside the lung is problematic. Nonetheless, K5CrePR1 may be ideal if genetic recombination is required in a high proportion of tracheal epithelial cells and extrapulmonary Cre activity is unimportant, for example in producing a culture of primary tracheal epithelial cells with a specific gene manipulation for in vitro studies or assessing susceptibility of the conducting airway epithelium to carcinogens after deletion of a tumor suppressor. In this study, we did not rigorously determine which tracheal epithelial cells express K5CrePR1 in the absence of induction or whether $\mathrm{K} 5 \mathrm{CrePR}$ driven recombination persists over months. K5CrePR1 causes genetic recombination in a higher proportion of the conducting airway epithelium than the induced K14CreER construct 5, 19 and appears to target a similar proportion of tracheal epithelial cells as the induced $\mathrm{K} 5 \mathrm{CreER}^{\mathrm{T} 2}$ construct $^{3}$.

K14CrePR1 has less RU486-independent activity than $\mathrm{K} 5 \mathrm{CrePR} 1$ and is inducible in a fraction of $\mathrm{K} 5$ positive basal cells by tracheal RU486. While K14CrePR1 is less active than K5CrePR1, this may be reflective of fewer K14 positive than $\mathrm{K} 5$ positive tracheal basal cells, however, the tissue distribution and tracheal activity of K14 directed Cre activity is similar to what was previously reported with K14CreER ${ }^{\mathrm{T}}$ after induction with systemic tamoxifen 5,18,19 and is consistent with the K14 expression observed in the murine trachea. When K14CreER ${ }^{\mathrm{T}}$ was used for lineage tracing basal airway cells, systemic tamoxifen was used for induction and ligand-independent Cre activity in the conducting airways was not reported 5, 19, making it difficult to directly compare K14CrePR1 and $\mathrm{K} 14 \mathrm{CreER}{ }^{\mathrm{T}}$. In any case, K14CrePR1 could potentially be used for evaluating lineage relationships between K14 positive tracheal cells and other cell types but would not be effective if experimental design required comprehensive alteration of gene expression in the entire $\mathrm{K} 5$ positive basal cell population. Our study suggests it may be possible to use a similar lipid delivery system for the 4-hydroxytamoxifen inducer of $\mathrm{K} 14 \mathrm{CreER}^{\mathrm{T}}$ or K5CreER ${ }^{\mathrm{T} 2}$ to achieve airway basal cell gene manipulation while minimizing Cre activity in other tissues. Although overall inducible Cre activity of $\mathrm{K} 5 \mathrm{Cre}{ }^{*} \mathrm{PR}$ is less than that of $\mathrm{K} 5 \mathrm{CrePR} 1$ or K14CrePR1, K5Cre*PR displays almost no ligand-independent Cre activity in the trachea or other keratin positive tissues (not shown). Induction with tracheal RU486 results in Cre activity in tracheal basal cells and Clara cells that increases with time and is consistent with targeting of basal cells that then give rise to additional basal cells, Clara cells, and ciliated epithelial cells as previously reported with the K5CreER ${ }^{\mathrm{T} 2}$ construct ${ }^{3}$. While K5Cre*PR would not be a good choice if experimental design required gene manipulation in the entire basal cell population, that $\mathrm{K} 5 \mathrm{Cre}{ }^{*} \mathrm{PR}$ is more tightly regulated makes it an attractive construct for use in cancer models where targeting of sporadic mutations would more closely mimic the somatic mutations leading to the development of human cancer. Our data also suggests that extra-pulmonary recombination can be minimized by tracheal RU486 delivery. Though this approach has not yet been reported in the lung, $\mathrm{K} 5 \mathrm{Cre}{ }^{*} \mathrm{PR}$ has been used to generate tumors in both the skin and oral epithelium via knock-in of K-ras ${ }^{\mathrm{G} 12 \mathrm{D}} 21,32$.

Presumably because of concerns regarding lipoid pneumonia or inflammation, reports of oil-based vehicles for lung delivery are limited. Although steroids are routinely delivered to the airways in clinical practice, this is typically done using an aerosol, an approach that is challenging in the experimental setting with very small quantities of material and when wanting to avoid delivery to the oropharynx or nasopharynx. One report demonstrated that a mixture of ethyl acetate/olive oil failed to deliver toluene diisocyanate (TDI) to the lung when administered by nasal inhalation ${ }^{33}$, while another report described repeated tracheal instillations with TDI in olive oil ${ }^{34}$. Although the second report demonstrated an increase in BAL eosinophils, inflammation, and goblet cell hyperplasia with TDI-olive oil instillation, the presence of LLM was not described ${ }^{34}$. We show that an acetone/sesame oil mixture can safely deliver RU486 to the mouse lung but causes a short-term inflammatory response comprised of neutrophils and LLM. Despite the inflammatory response demonstrated by survival BAL, there is minimal evidence of inflammation in the conducting airways or lung parenchyma (Supplemental Figure S1). While it cannot be excluded that inflammation contributes to Cre activation, Cre activity in the airways of vehicle treated and untreated K14CrePR1.Rosa26 mice was similar (not shown) and 
there is minimal Cre activity in vehicle treated K5Cre*PR.Rosa26 mice. Taken together, our data show that a single pulmonary dose of lipid/organic solvent is better tolerated than anticipated.

In the process of evaluating the response to tracheal instillation of potential RU486 vehicles, we developed a simple but potentially useful technique for performing survival BAL on non-ventilated mice. Although repeated BAL of individual ventilated mice has been reported 35 , the widespread use of this technique has likely been limited by the requirement for mechanical ventilation. Although the amounts of instilled and recovered lavage fluid are reduced compared to mechanically ventilated mice $(200 \mu$ instilled and $\sim 100 \mu 1$ recovered vs. $600 \mu \mathrm{l}$ instilled and $\sim 420 \mu \mathrm{l}$ recovered), our technique is simple and permits repeated sampling of individual animals over time, potentially reducing the number of animals required for a given study, and allowing each animal to serve as its own control. In addition, although we report an overall mortality of $8 \%$ for this technique, the mortality fell with increasing operator experience. This approach could be particularly useful for studies examining recovery of infectious agents or BAL cellular profiles in response to various stimuli even though the amount of recovered BAL fluid may limit some downstream applications.

In summary we developed a method for delivering lipid soluble Cre activators to the lung that is safe and effective and can be used to minimize extra-pulmonary Cre recombinase activity when using keratin driven Cre constructs or other inducible Cre constructs targeted by broadly expressed promoters. These approaches should facilitate the modeling of diseases thought to arise from airway basal cells such as lung squamous cell carcinoma.

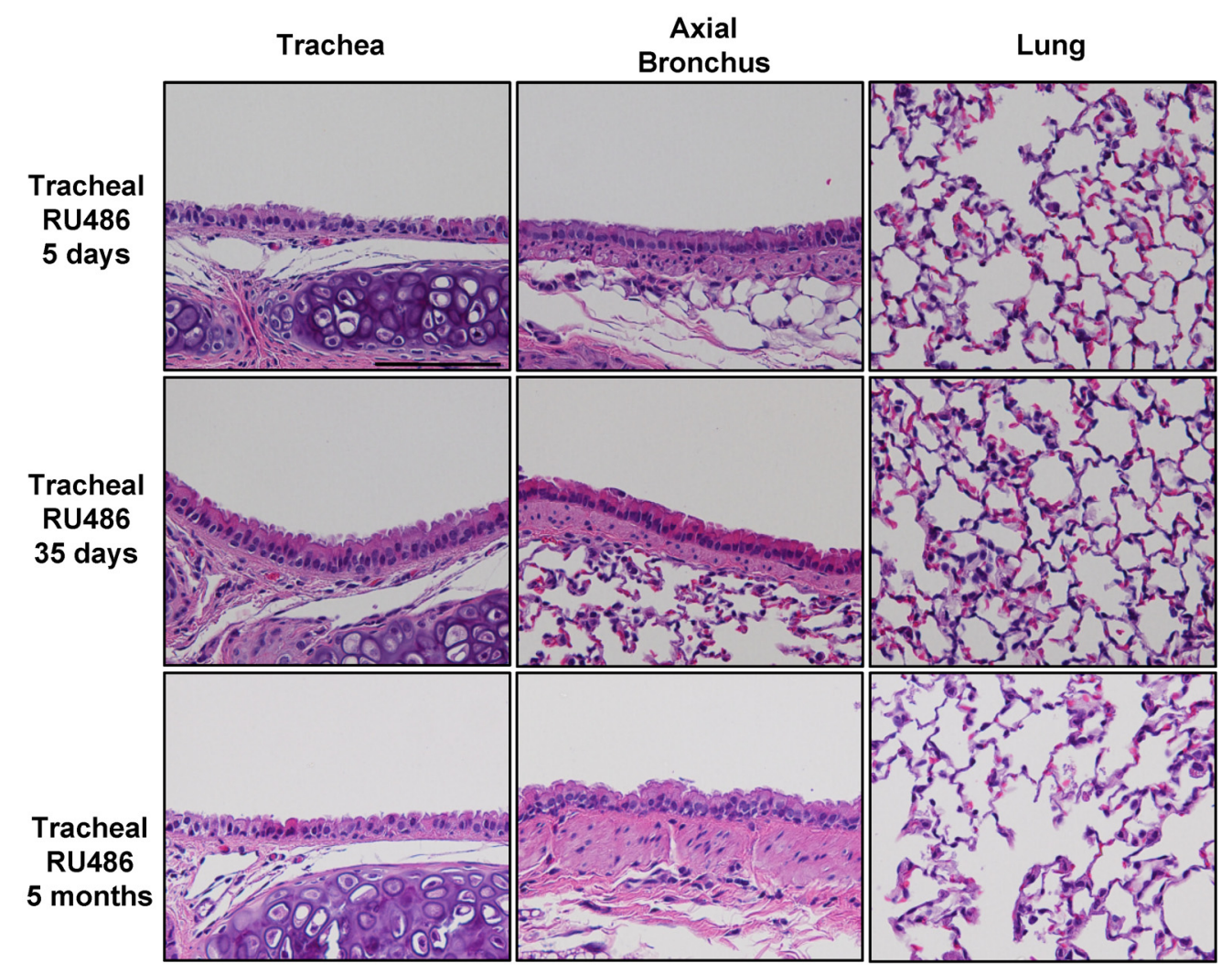

Supplemental Figure SI: Hematoxylin and eosin stained sections of the trachea, axial bronchus and lung parenchyma 5 days, 5 weeks, and 5 months after instillation of $500 \mu \mathrm{g}$ RU 486 in $20 \mu \mathrm{l}$ of $10 \%$ acetone $/ 90 \%$ sesame oil showing no obvious inflammation or morphological abnormality. The scale bar represents $100 \mu \mathrm{m}$.

\section{Acknowledgments}

We thank Dr. David Jacoby for providing human tracheal sections. This work was supported by the NIH/NCI (F32 CA119467 and K08 CA131483 to
S.P.M.) and NIH/NIDCR (R01 DE015953 to X.J.W.). S.P.M. was also supported by T32-CA 106195 ("Training in Skin Pathobiology") and the Oregon Medical Research Foundation. 


\section{Conflicts of interest}

The authors report no conflict of interest. The authors alone are responsible for the content and writing of this manuscript.

\section{References}

1. Perl AK, Wert SE, Loudy DE, Shan Z, Blair PA, Whitsett JA. Conditional recombination reveals distinct subsets of epithelial cells in trachea, bronchi, and alveoli. Am J Respir Cell Mol Biol 2005;33:455-62.

2. Kim CF, Jackson EL, Woolfenden $\mathrm{AE}$, et al. Identification of bronchioalveolar stem cells in normal lung and lung cancer. Cell 2005;121:823-35.

3. Rock JR, Onaitis MW, Rawlins EL, et al. Basal cells as stem cells of the mouse trachea and human airway epithelium. Proc Natl Acad Sci U S A 2009;106:12771-5.

4. Rawlins EL, Okubo T, Xue Y, et al. The role of Scgb1a1+ Clara cells in the long-term maintenance and repair of lung airway, but not alveolar, epithelium. Cell Stem Cell 2009;4:525-34.

5. Hong KU, Reynolds SD, Watkins S, Fuchs E, Stripp BR. Basal cells are a multipotent progenitor capable of renewing the bronchial epithelium. Am J Pathol 2004;164:577-88.

6. Borthwick DW, Shahbazian M, Krantz QT, Dorin JR, Randell $\mathrm{SH}$. Evidence for stem-cell niches in the tracheal epithelium. Am J Respir Cell Mol Biol 2001;24:662-70.

7. Engelhardt JF, Schlossberg H, Yankaskas JR, Dudus L. Progenitor cells of the adult human airway involved in submucosal gland development. Development 1995;121:2031-46.

8. Li H, Cho SN, Evans CM, Dickey BF, Jeong JW, DeMayo FJ. Cre-mediated recombination in mouse Clara cells. Genesis 2008;46:300-7.

9. Bertin G, Poujeol C, Rubera I, Poujeol P, Tauc M. In vivo Cre/loxP mediated recombination in mouse Clara cells. Transgenic Res 2005;14:645-54.

10. Ji H, Houghton AM, Mariani TJ, et al. K-ras activation generates an inflammatory response in lung tumors. Oncogene 2006;25:2105-12.

11. Zhang Y, Huang G, Shornick LP, et al. A transgenic FOXJ1-Cre system for gene inactivation in ciliated epithelial cells. Am J Respir Cell Mol Biol 2007;36:515-9.

12. Jackson EL, Willis N, Mercer K, et al. Analysis of lung tumor initiation and progression using conditional expression of oncogenic K-ras. Genes Dev 2001;15:3243-8.

13. Clark JC, Tichelaar JW, Wert SE, et al. FGF-10 disrupts lung morphogenesis and causes pulmonary adenomas in vivo. Am J Physiol Lung Cell Mol Physiol 2001;280:L705-15.

14. Tichelaar JW, Lu W, Whitsett JA. Conditional expression of fibroblast growth factor-7 in the developing and mature lung. J Biol Chem 2000;275:11858-64.

15. Iwanaga K, Yang Y, Raso MG, et al. Pten inactivation accelerates oncogenic K-ras-initiated tumorigenesis in a mouse model of lung cancer. Cancer Res 2008;68:1119-27.

16. Yanagi S, Kishimoto H, Kawahara K, et al. Pten controls lung morphogenesis, bronchioalveolar stem cells, and onset of lung adenocarcinomas in mice. J Clin Invest 2007;117:2929-40.

17. Ji H, Ramsey MR, Hayes DN, et al. LKB1 modulates lung cancer differentiation and metastasis. Nature 2007;448:807-10.

18. Vasioukhin V, Degenstein L, Wise B, Fuchs E. The magical touch: genome targeting in epidermal stem cells induced by tamoxifen application to mouse skin. Proc Natl Acad Sci U S A 1999;96:8551-6.

19. Hong KU, Reynolds SD, Watkins S, Fuchs E, Stripp BR. In vivo differentiation potential of tracheal basal cells: evidence for multipotent and unipotent subpopulations. Am J Physiol Lung Cell Mol Physiol 2004;286:L643-9.
20. Caulin C, Nguyen T, Longley MA, Zhou Z, Wang XJ, Roop DR. Inducible activation of oncogenic K-ras results in tumor formation in the oral cavity. Cancer Res 2004;64:5054-8.

21. Lu SL, Herrington $H$, Reh D, et al. Loss of transforming growth factor-beta type II receptor promotes metastatic head-and-neck squamous cell carcinoma. Genes Dev 2006;20:1331-42.

22. Kellendonk C, Tronche F, Monaghan AP, Angrand PO, Stewart F, Schutz G. Regulation of Cre recombinase activity by the synthetic steroid RU 486. Nucleic Acids Res 1996;24:1404-11.

23. Zhou Z, Wang D, Wang XJ, Roop DR. In utero activation of K5.CrePR1 induces gene deletion. Genesis 2002;32:191-2.

24. Berton TR, Wang XJ, Zhou Z, et al. Characterization of an inducible, epidermal-specific knockout system: differential expression of lac $Z$ in different $C r e$ reporter mouse strains. Genesis 2000;26:160-1.

25. Wunderlich FT, Wildner H, Rajewsky K, Edenhofer F. New variants of inducible Cre recombinase: a novel mutant of Cre-PR fusion protein exhibits enhanced sensitivity and an expanded range of inducibility. Nucleic Acids Res 2001;29:E47.

26. Soriano P. Generalized lacZ expression with the ROSA26 Cre reporter strain. Nat Genet 1999;21:70-1.

27. Brown RH, Walters DM, Greenberg RS, Mitzner W. A method of endotracheal intubation and pulmonary functional assessment for repeated studies in mice. J Appl Physiol 1999;87:2362-5.

28. Boers JE, Ambergen AW, Thunnissen FB. Number and proliferation of basal and parabasal cells in normal human airway epithelium. Am J Respir Crit Care Med 1998;157:2000-6.

29. Pack RJ, Al-Ugaily LH, Morris G. The cells of the tracheobronchial epithelium of the mouse: a quantitative light and electron microscope study. J Anat 1981;132:71-84.

30. Gondouin A, Manzoni P, Ranfaing E, et al. Exogenous lipid pneumonia: a retrospective multicentre study of 44 cases in France. Eur Respir J 1996;9:1463-9.

31. Bornstein S, White R, Malkoski S, et al. Smad4 loss in mice causes spontaneous head and neck cancer with increased genomic instability and inflammation. $J$ Clin Invest 2009;119:3408-19.

32. Caulin C, Nguyen T, Lang GA, et al. An inducible mouse model for skin cancer reveals distinct roles for gain- and loss-of-function p53 mutations. J Clin Invest 2007;117:1893-901.

33. Ebino K, Lemus R, Karol MH. The importance of the diluent for airway transport of toluene diisocyanate following intranasal dosing of mice. Inhal Toxicol 1999;11:171-85.

34. Ban M, Morel G, Langonne I, Huguet N, Pepin E, Binet S. TDI can induce respiratory allergy with Th2-dominated response in mice. Toxicology 2006;218:39-47.

35. Walters DM, Wills-Karp M, Mitzner W. Assessment of cellular profile and lung function with repeated bronchoalveolar lavage in individual mice. Physiol Genomics 2000;2:29-36. 